\title{
LECTOTIPIFICACIONES Y NOVEDAD TAXONÓMICA EN SPIGELIA (LOGANIACEAE) PARA LA FLORA DEL CONO SUR
}

\author{
Christian A. Zanotti \\ Instituto de Botánica Darwinion (CONICET-ANCEFN), Labardén 200, Casilla de Correo 22, B1642HYD, San Isidro, \\ Buenos Aires, Argentina; czanotti1979@gmail.com (autor corresponsal).
}

\begin{abstract}
Zanotti, C. A. 2021. Lectotypifications and taxonomic novelty in Spigelia (Loganiaceae) for the flora of the Southern Cone. Darwiniana, nueva serie 9(2): 265-272.

In the framework of the taxonomic treatment of the family Loganiaceae for the Argentinean Flora, it was necessary to lectotypify 12 names described for the genus Spigelia. On the other hand, Spigelia beccabungoides, cited as endemic to the Flora of Paraguay is synonymized under Justicia comata (Acanthaceae).
\end{abstract}

Keywords. Acanthaceae; floristics; Justicia; Loganiaceae; Paraguay; Spigelia; typification.

Resumen. Zanotti, C. A. 2021. Lectotipificaciones y novedad taxonómica en Spigelia (Loganiaceae) para la flora del Cono Sur. Darwiniana, nueva serie 9(2): 265-272.

En el marco del tratamiento taxonómico de la familia Loganiaceae para la Flora Argentina, se encontró la necesidad de lectotipificar 12 nombres descritos para el género Spigelia. Por otro lado, se sinonimiza a Spigelia beccabungoides, especie citada como endémica de la Flora del Paraguay, bajo Justicia comata (Acanthaceae).

Palabras clave. Acanthaceae; florística; Justicia; Loganiaceae; Paraguay; Spigelia; tipificación.

\section{INTRODUCCIÓN}

Spigelia L. (Loganiaceae) es un género neotropical con 60-85 especies que se distribuyen desde el sur de los Estados Unidos de América hasta la Argentina y Chile, con su centro de diversidad en el este del Brasil (Fernández Casas, 2001; Islas Hernández et al., 2017; Struwe et al., 2018; Zanotti, 2020). Sus representantes comprenden hierbas anuales, perennes o sufrútices, de hojas generalmente opuestas o dispuestas en pseudoverticilos tetrámeros en el ápice de las ramas y con estípulas interpeciolares soldadas; las inflorescencias son cimas escorpioides o espigas unilaterales terminales y axilares, a veces reducidas a una o tres flores perfectas, pentámeras y gamopétalas; los frutos son cápsulas loculicidas de dehiscencia circuncisa basal (Hurley, 1967;
Gould, 1997; Fernández Casas, 2001; Popovkin et al., 2011; Struwe et al., 2018; Zanotti, 2020). Spigelia es un género monofilético y forma una tribu monotípica dentro de la familia, aunque las relaciones filogenéticas entre las especies aún son poco conocidas (Popovkin et al., 2011; Struwe et al., 2018). Aunque Spigelia ha sido estudiado en revisiones taxonómicas y nomenclaturales (Hurley, 1967; Bravo, 1971; Gould, 1997; Fernández Casas, 2016), se encontró la necesidad de tipificar 12 nombres en el marco del tratamiento taxonómico de la familia Loganiaceae para la Flora Argentina (Zanotti, 2020). Por otro lado, se sinonimiza a Spigelia beccabungoides Kraenzl., especie erróneamente descripta para la familia Loganiaceae y conocida hasta el momento como endémica de la Flora del Paraguay, bajo Justicia comata (L.) Lam. (Acanthaceae). 


\section{MATERIALES Y MÉTODOS}

Se siguieron técnicas convencionales de taxonomía. Los tipos nomenclaturales fueron examinados a través de imágenes digitales de alta calidad de ejemplares de los herbarios A, BM, C, F, G, GH, GOET, HAL, K, L, M, MICH, MO, MPU, NY, P, S, UC, US, disponibles en JSTOR (https:// plants.jstor.org), excepto por los ejemplares Hassler 6145, 7746, 8294 y $8294 a$, que fueron enviados digitalmente por el personal del herbario G. Los acrónimos de los herbarios se abreviaron de acuerdo con Thiers (2021). En esta contribución, la lista de sinónimos sólo contiene a los nombres para los cuales se designa lectotipo aquí. Para una sinonimia completa véase Zanotti (2020).

\section{TRATAMIENTO TAXONÓMICO}

Spigelia caaguazuensis Kraenzl., Repert. Spec. Nov. Regni Veg. 14: 293. 1916. TIPO: Paraguay, Caaguazú, "in campis", II-1905, E. Hassler 8951 (lectotipo G 0381326 [3 pliegos] [Hassler]! aquí designado; isolectotipos BM 000089818!, C 10014005!, G 00381323 [2 pliegos] [Barbey-Boisser]!, G 00381283 [Delessert]!, GH 00076706!, K 000573366!, MICH 1192933!, MO 391999!, MPU 018329!, NY 00297391!, P 00506397!, P 00506398!, S-R-5844!, US 00112908 [fragmento]!).

Distribución y hábitat. Spigelia caaguazuensis se distribuye en el sur del Brasil, Paraguay y nordeste de Argentina (Misiones). Se la ha coleccionado en campos abiertos con suelos secos.

Observaciones. En el protólogo de Spigelia caaguazuensis, Kraenzlin (1916) citó dos sintipos: "Prope Caaguazu in campis, Hassler no. 8951, $8951 \mathrm{a}$ ". Ambos se hallan depositados en G, ya que Kraenzlin publicó este nombre entre las novedades del herbario Hassler, como lo indica el título de la obra "Ex herbario Hassleriano: Novitates paraguarienses". Cabe destacar, que el herbario personal de Hassler fue donado al herbario G luego del fallecimiento del mismo (Ramella et al., 2008). Ambos sintipos coinciden con la descripción del protólogo y son ejemplares completos.
No obstante, se elige el sintipo Hassler 8951 por presentar más duplicados depositados en diferentes herbarios que el sintipo remanente. Se designa como lectotipo al espécimen que presenta el código de barras G 00381326 montado en 3 pliegos, y que posee la etiqueta original proveniente del herbario Hassler y un sello que lleva la palabra "unicum".

Spigelia nicotianiflora Chodat \& Hassl. var. nicotianiflora, Bull. Herb. Boissier, sér. 2, 3: 917. 1903. TIPO: no designado.

Spigelia nicotianiflora Chodat \& Hassl. var. capibarensis Chodat \& Hassl., Bull. Herb. Boissier, sér. 2, 3: 918. 1903. TIPO: Paraguay, Caaguazú, "In arenosis pr. Vaqueria Capibary", IX-18981899, E. Hassler 4421 (lectotipo G 00381349! aquí designado; isolectotipo BM 000757912 [las dos plantas a la derecha del pliego, mezclada con el tipo de la var. puberula, Hassler 8269]!, G 00381348 [Barbey-Boisser]!, G 00381350 !, K 000573344!, NY 00564025!, NY 00564026!, P 00507617!, P 00507618!, UC 944159!).

Distribución y hábitat. Spigelia nicotianiflora se distribuye en Brasil, Paraguay y nordeste de la Argentina (Corrientes), donde crece en campos arenosos. La especie ha sido citada para el centro y sur de México en diferentes tratamientos taxonómicos; no obstante, fue excluida recientemente de la flora mexicana (Islas Hernández et al., 2017).

Observaciones. Siguiendo el Art. 60.10, Ej. 36 del Código Internacional de Nomenclatura para algas, hongos y plantas (ICN; Turland et al., 2018), la ortografía correcta para el epíteto específico de este taxón es "nicotianiflora" y no "nicotianaeflora", como fue publicado en el protólogo (Chodat \& Hassler, 1903).

Cuando Chodat \& Hassler (1903) describieron a Spigelia nicotianiflora, no indicaron material tipo, pero sí lo hicieron para las dos variedades que describieron bajo este nombre (var. capibarensis y var. puberula). Ambos nombres varietales fueron válidamente publicados, ya que la descripción original de la especie abarca las circunscripciones de ambas variedades (Art. 26.2 del ICN; Turland et al., 2018). Hurley (1967: 45) indicó como tipo de 
Spigelia nicotianiflora var. nicotianiflora material ajeno al protólogo (véase Art. 26.2, Ej. 3 y Ej. 4 del ICN, Turland et al., 2018), la colección Rojas 9913 depositada en BM y G (con 4 pliegos) y con fotografías de la serie del Field Museum. Por otro lado, Gould (1997: 174) lectotipificó a este nombre utilizando esta misma colección, con el espécimen depositado en $\mathrm{G}$ (número 159) pero, según el Art. 30.9 del ICN (Turland et al., 2018), la misma no se puede considerar una lectotipificación efectiva. Por último, Fernández Casas (2016: 30) reiteró esta información, pero no designó ningún espécimen como lectotipo de la variedad típica. Desafortunadamente, ninguno de estos especímenes, ni otro ejemplar que se ajuste a la descripción de la variedad típica ha podido ser estudiado para la eventual designación de un lectotipo. Sólo se pudo verificar la existencia de la colección Rojas 9913 por una foto localizada en la página del Field Museum (foto $\mathrm{N}^{\circ} 3925$ de la serie; https://collections-botany. fieldmuseum.org/catalogue/237459). Por lo tanto, $S$. nicotianiflora var. nicotianiflora continúa siendo un nombre válido sin tipo designado.

En el protólogo de Spigelia nicotianiflora var. capibarensis, Chodat \& Hassler (1903) citaron "in arenosis pr. Vaqueria Capibary, Sept., n. 4421". Se encontraron 3 especímenes de la colección Hassler 4421 depositados en $\mathrm{G}$, donde se hallan los materiales de Hassler coleccionados en Paraguay (Ramella et al., 2008) y donde trabajaban Chodat \& Hassler (Stafleu \& Cowan, 1976; 1979), con lo cual se presume que fueron oportunamente estudiados en $\mathrm{G}$ por Chodat \& Hassler. Se elige como lectotipo al espécimen que lleva el código de barras G 00381349, porque coincide con la descripción del protólogo, es un ejemplar completo y porque presenta la etiqueta original con la observación "nob" al final del nombre del taxón, que también se observa en el protólogo.

Spigelia paraguariensis Chodat, Bull. Herb. Boissier, sér. 2, 1: 408. 1901. TIPO: Paraguay, Cordillera, "Ad marginem silvae pr. Piribebuy", II-1885-1895, E. Hassler 1874 (lectotipo G 00381356 ! aquí designado; isolectotipos BM 000089812 !, G 00381357 [2 pliegos] [BarbeyBoisser]!, G 00381365!, NY 00297397!, P 00507620!, P 00507621!).
Spigelia paraguariensis Chodat f. subscandens Chodat \& Hassl., Bull. Herb. Boissier, sér. 2, 3: 917. 1903. TIPO: Paraguay, Cordillera, "Ad marginem silvae Tobaty”, IX-1900, E. Hassler 6145 (lectotipo G 00394238 [Chodat]! aquí designado; isolectotipos G 00392435 [Hassler]!, G 00392441 [Delessert]!, G 00442817 [BarbeyBoisser]!, GH 00107110!, MPU 013247!, P 00507625!, P 00507626!, S 04-26!, UC 944157!; isolectotipo probable BM 000757907 p.p. [planta a la derecha del pliego]!).

Spigelia epilobioides Kraenzl., Bot. Jahrb. Syst. 40: 306. 1908. TIPO: Paraguay, Cordillera, "Cerros Tobaty: Cerro Penitente", 15-I-1903, K. A. G. Fiebrig 753 (lectotipo BM 000089804! aquí designado; isolectotipos F 0062137F!, F 0062138F [fragmento]!, GH 00107103!, GOET 005451!, K 000573365!, L 0005197!, M 0089517!, US 00112911!).

Distribución y hábitat. Spigelia paraguariensis se distribuye en Bolivia, Paraguay y en el nordeste de la Argentina (Misiones), en donde se la halla en claros o márgenes de la selva.

Observaciones. En el protólogo de Spigelia paraguariensis, Chodat (1901) citó "Ad marginem silvae p. Péribebuy, Febr., 1874". Se encontraron 3 especímenes correspondientes a la colección Hassler 1874 depositados en G. Allí trabajaba Chodat (Stafleu \& Cowan, 1976), por lo tanto, se sospecha que Chodat basó su diagnosis en material depositado en G. Por otro lado, los materiales de Hassler coleccionados en Paraguay se hallan en $\mathrm{G}$ (Ramella et al., 2008). Se elige como lectotipo al espécimen G 00381356 porque concuerda con el protólogo y es un ejemplar completo. Además, este espécimen, presenta la etiqueta original con el nombre de la especie escrito con la letra manuscrita de Chodat y posee más cantidad de flores en antesis.

En el protólogo de Spigelia paraguariensis f. subscandens, Chodat \& Hassler (1903) citaron "ad marginem silvae pr. Tobaty, Sept., n. 6145". Los materiales originales colectados por Hassler en $\mathrm{Pa}$ raguay se hallan depositados en $\mathrm{G}$ (Ramella et al., 2008). Por otro lado, tanto Chodat como Hassler trabajaron en $\mathrm{G}$, y la diagnosis del taxón presumiblemente fue realizada en base a los materiales depositados en este herbario (Stafleu \& Cowan, 1976; 1979). 
En el herbario $\mathrm{G}$ se encontraron 4 especímenes de la colección Hassler 6145. Se elige como lectotipo al espécimen con el código de barras G 00394238 , proveniente del herbario Chodat, que presenta la etiqueta original de la colección y coincide con lo descripto en el protólogo.

En el protólogo de Spigelia epilobioides, Kraenzlin (1908) citó "Paraguay: Cerros de Tobati, am Cerro Penitente, auf purem (sic) Stein (Fiebrig n. 753!). El material original fue depositado en B, hecho que se ratifica por la fotografía de la serie del Field Museum (foto $\mathrm{N}^{\circ} 3916$; https://collectionsbotany.fieldmuseum.org/catalogue/237434) en la que se observa un labelo de 1906 con la letra manuscrita de Kraenzlin donde se indica el nombre de la especie. De acuerdo con Hiepko (1987), las colecciones de B previas a 1943 correspondientes a la familia Loganiaceae, se perdieron durante la destrucción del herbario en el curso de la Segunda Guerra Mundial. Por esta razón, se elige como lectotipo el espécimen depositado en BM, ya que presenta más cantidad de flores en antesis en relación a los otros duplicados, y se halla en un buen estado de conservación.

Spigelia scabra Cham. \& Schltdl., Linnaea 1(2): 202. 1826. TIPO: Uruguay, Montevideo, "Brasilia, in provincia Cisplatina", s.d., F. Sellow s.n. (lectotipo HAL 0069906! aquí designado).

Spigelia hassleriana Chodat, Bull. Herb. Boissier, sér. 2, 1: 407. 1901. TIPO: Paraguay, Cordillera, "Cordillera de Altos, in campo" VIII-18851895, E. Hassler 824 (lectotipo G 00381335 ! aquí designado; isolectotipos G 00381336 !, G 00381337 [Barbey-Boisser]!, K 000573346 !, P 00507566!, P 00507567!).

Spigelia hassleriana Chodat var. major Chodat \& Hassl., Bull. Herb. Boissier, sér. 2, 3: 916. 1903. TIPO: Paraguay, In silva Pacoba, Flumin. Corrientes”, IX-1898-1899, E. Hassler 4507 (lectotipo G 00381347! aquí designado; isolectotipos BM 000089821!, G 00381344 [2 pliegos] [Barbey-Boisser]!, G 00381345 [Delessert]!, G 00381346!, K 000573345!, NY 00297396!, P 00507562!, P 00507563!, UC 950109!).
Spigelia guaranitica Chodat \& Hassl., Bull. Herb. Boissier, sér. 2, 3: 917. 1903. TIPO: Paraguay, Amambay, "in regione cursus superioris fluminis Apa", XII-1901-1902, E. Hassler 8063 (lectotipo G 00381284 [2 pliegos]! aquí designado; isolectotipos A 00107108 [2 pliegos]!, BM 000089805!, C 10014015!, G 00381324 [Delessert]!, G 00381325 [2 pliegos] [Barbey-Boisser]!, G 00381338 [2 pliegos]!, K 000573361!, MICH 1191519!, MPU 013246!, NY 00297395!，P 00507560!，P 00753826!, S-R-5845!, UC 944161!).

Spigelia hassleriana Kraenzl., Bot. Jahrb. Syst. 40: 307. 1908, nom. illeg., non Chodat 1901. TIPO: Paraguay, "Paraguaria centralis", 1897, E. Hassler 2132 (lectotipo BM 000089820 ! aquí designado; isolectotipo $\mathrm{K}$ 000573347!).

Spigelia breviflora (Chodat \& Hassl.) H. Hurley ex Fern. Casas, Fontqueria 55(5): 27. 2001. Spigelia humboldtiana Cham. \& Schltdl. var. breviflora Chodat \& Hassl., Bull. Herb. Boissier, sér. 2, 3: 916. 1903. TIPO: Paraguay, Amambay, "In regione calcarea cursus superioris fluminis Apa, in silvis", XII-1901-1902, E. Hassler 7746 (lectotipo G 002229999 [3 pliegos] [Hassler]! aquí designado; isolectotipos BM 000089807!, G 0022229998 [Chodat]!, G 00230000 [BarbeyBoissier]!, K 000573374!, MO 694074!, NY 00563987!， P 00506394!， S-R-5847!, UC 944160 !).

Distribución y hábitat. Spigelia scabra es una especie bastante polimorfa de una amplia distribución desde México hasta el centro de la Argentina. Crece en lugares húmedos a anegados, a orilla de ríos y arroyos de claros de bosques o selvas y también se la encuentra en ambientes disturbados.

Observaciones. En el protólogo de Spigelia scabra, Chamisso \& Schlechtendal (1826) citaron "In provincia Cisplatina Brasiliae legit Sellow". El material original fue depositado en B, lo cual se corrobora por la fotografía tomada del ejemplar tipo de la serie del Field Museum (F 0BN003927!; https://collections-botany.fieldmuseum.org/catalogue/ 237467) y que presenta la letra manuscrita del propio Schlechtendal con el nombre de la especie. 
De acuerdo con Hiepko (1987), las colecciones de B previas a 1943 correspondientes a la familia Loganiaceae, se perdieron durante la destrucción del herbario en el curso de la Segunda Guerra Mundial. Aquí se designa como lectotipo al espécimen depositado en HAL, ya que numerosos duplicados de los ejemplares tipo descritos entre 1819 y 1833 en B por Chamisso y Schlechtendal se transfirieron al herbario HAL (Braun \& Wittig, 2003). Por otro lado, el ejemplar depositado en HAL, presenta una etiqueta idéntica a la que posee el fototipo de F.

En el protólogo de Spigelia hassleriana, Chodat (1901) citó "In campo p. Cordill. de Altos, Aug., 824". Se encontraron 3 especímenes correspondientes a la colección Hassler 824 depositados en G. Allí trabajaba Chodat (Stafleu \& Cowan, 1976), por lo tanto, se sospecha que Chodat basó su diagnosis en material depositado en G. Por otro lado, los materiales de Hassler coleccionados en Paraguay se hallan en G (Ramella et al., 2008). Se elige como lectotipo al espécimen G 00381335 , ya que coincide con el protólogo, es un ejemplar completo, y presenta la etiqueta original de la colección con la observación dada en el protólogo "Spigelia nova aff. S. brachystachyae sed minor" con la letra manuscrita de Chodat.

En el protólogo de Spigelia hassleriana var. major, Chodat \& Hassler (1903) citaron dos sintipos: "in dumetis pr. Limpio, Nov., n. 3459; in silva Pacoba, (flumen Corrientes) Sept. N. 4507". A fines de lectotipificar el nombre en cuestión, se prefiere la colección Hassler 4507, ya que se encuentra representada en más de un herbario, contrariamente a lo que ocurre con el sintipo Hassler 3459, que solo se encuentra un pliego en el herbario G (G 00381328 [unicum]!). De la colección elegida, existen 4 especímenes depositados en $\mathrm{G}$, de los cuales se designa como lectotipo al que presenta el código de barras $\mathrm{G}$ 00381347. Este coincide con el protólogo, es un ejemplar completo, y lleva la etiqueta original con la observación "nob" al final del nombre del taxón, que también se observa en el protólogo. El nombre Spigelia guaranitica Chodat \& Hassl. var. interrupta publicado por Kraenzlin (1916), es un nombre superfluo de acuerdo con el Art. 52.1 del ICN (Turland et al., 2018), ya que el autor incluyó los sintipos mencionados por Hassler en el protólogo de S. hassleriana var. major.
En el protólogo de Spigelia guaranitica, Chodat \& Hassler (1903) citaron "in silvis apricis in regione cursus superioris fluminis Apa, Dec., n. 8063". Los materiales originales colectados por Hassler en Paraguay se hallan depositados en G (Ramella et al., 2008). Por otro lado, tanto Chodat como Hassler trabajaron en G, y se presume que la diagnosis del taxón se realizó en base a los materiales depositados en este herbario (Stafleu \& Cowan, 1976; 1979). Se encontraron 4 especímenes de esta colección depositados en G. En este marco, se elige como lectotipo al espécimen G 00381284 (montado en 3 pliegos) porque coincide con el protólogo, es un ejemplar completo, presenta la etiqueta original y posee un labelo con las medidas de las hojas y la cantidad de flores utilizados por Chodat \& Hassler para realizar la descripción de la especie. Además, el ejemplar lleva adjunto un dibujo con detalles de la flor realizados por el propio Chodat.

En el protólogo del homónimo posterior ilegítimo (Art. 53.1 del ICN; Turland et al., 2018) de Spigelia hassleriana (Kraenzlin, 1908) se cita "Zentral-Paraguay (Dr. Hassler n. 2132)", sin consignar el herbario donde se halla dicha colección. El ejemplar tipo descripto por Kraenzlin presumiblemente fue depositado en B, como ocurre en el caso de Spigelia epilobioides descripta en la misma publicación por este mismo autor, y considerando también que el herbario personal y los tipos descriptos por Kraenzlin fueron adquiridos por B en 1907 (Stafleu \& Cowan, 1979). De acuerdo con Hiepko (1987), las colecciones de B previas a 1943 correspondientes a la familia Loganiaceae, se perdieron durante la destrucción del herbario en el curso de la Segunda Guerra Mundial y, lamentablemente, no existe foto de la serie del Field Museum para ratificar su presencia en este herbario. En el herbario G, donde se hallan los materiales de Hassler coleccionados en Paraguay (Ramella et al., 2008), tampoco se ha podido localizar este ejemplar. Por este motivo, se lectotipifica con el ejemplar depositado en BM, por estar en un mejor estado de conservación que el duplicado depositado en K.

En el protólogo de Spigelia humboldtiana var. breviflora, Chodat \& Hassler (1903) citaron "in silvis in regione cursus superioris fluminis Apa, Nov., n. 7746". Los materiales originales 
colectados por Hassler en Paraguay, se hallan depositados en G (Ramella et al., 2008). Por otro lado, tanto Chodat como Hassler trabajaron en G (Stafleu \& Cowan, 1976; 1979) y, por lo tanto, se presume que la diagnosis del taxón fue realizada en base a los materiales depositados en este herbario. Se encontraron 3 especímenes de la colección Hassler 7746 depositados en el herbario G. El espécimen elegido como lectotipo coincide con el protólogo, es un ejemplar completo, y se encuentra montado en 3 pliegos que originalmente pertenecieron al herbario Hassler. El pliego de este espécimen que presenta la etiqueta original, posee la inscripción "nob" al final de nombre del taxón con la letra manuscrita de Hassler como figura en el protólogo.

\section{Nuevo sinónimo para la flora del Paraguay}

Justicia comata (L.) Lam., Encycl. 1: 632. 1785. Dianthera comata L., Syst. Nat. Ed. 10, 2: 850. 1759. TIPO: Jamaica, sin localidad consignada, P. Browne s.n. (lectotipo LINNHL29-2! designado por V. A. W. Graham, Kew Bull. 34: 618. 1988).

Spigelia beccabungoides Kraenzl., Bot. Jahrb. Syst. 40: 307. 1908. TIPO: Paraguay, Amambay, "in regione cursus superioris fluminis Apa", XII-1901-1902, E. Hassler 8294 (lectotipo G 00102276 [2 pliegos] [Hassler]! aquí designado; isolectotipos G 00102277 [2 pliegos] [Chodat]!, G 00102278 [Delessert]!, K 000529184!), syn. nov.

Distribución y hábitat. Justicia comata es una especie ampliamente distribuida en América tropical desde el sur de México hasta Bolivia, Paraguay y el norte de la Argentina, en donde habita lugares con suelos anegados o muy húmedos.

Observaciones. Desde su descripción hasta la actualidad, Spigelia beccabungoides ha sido considerada como la única especie endémica de la familia Loganiaceae citada para la flora del Paraguay (Peña Chocarro \& De Egea, 2018; Zuloaga et al., 2019). En el protólogo de esta especie, Kraenzlin (1908) citó dos sintipos "Dr. Hassler n. 8284! und 8294a!". La colección Hassler 8284 citada en el protólogo corresponde a un error tipográfico, ya que este número de colección corresponde al holotipo de Ipomoea bonariensis Hook. f. glabrata Hassl. (= Ipomoea bonariensis, Convolvulaceae, G 00174738!). Este hecho se ratifica ya que, en el herbario K en donde se hallan algunos de los tipos determinados por Kraenzlin (Stafleu \& Cowan, 1979), se encontraron las colecciones Hassler 8294 y $8294 a$ bajo el nombre de $S$. beccabungoides. Por otro lado, cuando el autor realizó un breve comentario en el protólogo acerca del hábito de esta especie, los números de estas dos colecciones se encuentran bien tipografiados. En el protólogo de $S$. beccabungoides no se consignó el herbario donde se hallan depositados los sintipos, pero presumiblemente fueron depositados en $\mathrm{B}$, ya que el herbario personal y los tipos descriptos por Kraenzlin fueron adquiridos por B en 1907 (Stafleu \& Cowan, 1979). De acuerdo con Hiepko (1987), las colecciones de B previas a 1943 correspondientes a la familia Loganiaceae, se perdieron durante la destrucción del herbario en el curso de la Segunda Guerra Mundial. Lamentablemente, no existe evidencia de que el material descripto por Kraenzlin se halle en dicho herbario, ya que tampoco se cuenta con un fototipo de F, como sí ocurre en el caso Spigelia epilobioides descripta en la misma publicación por el mismo autor. Por otro lado, en el herbario $\mathrm{G}$ donde se hallan los materiales de Hassler coleccionados en Paraguay (Ramella et al., 2008), se encontraron especímenes que se ajustan al protólogo. Ninguno de ellos presenta el nombre de la especie y tampoco se encuentran anotados por Kraenzlin, con lo cual es posible que estos ejemplares no hayan sido estudiados por este autor. De todas maneras, a fines de lectotipificar el nombre en cuestión, se prefiere la colección Hassler 8294, ya que presenta una mayor cantidad de duplicados que la colección remanente Hassler 8294a. De la colección seleccionada, se elige como lectotipo al espécimen G 00102276 (montado en 2 pliegos), ya que se ajusta a la descripción del protólogo y es un ejemplar completo.

Hurley (1967: 195) sólo analizó la descripción original de $S$. beccabungoides y la trató como un 
taxón dudoso ante la imposibilidad de examinar el material original. Por otro lado, la forma y el tamaño de las hojas le sugirieron una relación estrecha con Spigelia scabra Cham. \& Schltdl., pero afirmó que el menor tamaño de sus flores la aleja de dicha especie. Posteriormente, Fernández Casas (2016: 47) también mantuvo a esta especie como dudosa y aseveró que se trata de un representante de la familia Lamiaceae, según un espécimen examinado en W. $\mathrm{Al}$ estudiar los materiales originales y el protólogo de $S$. beccabungoides, se confirma aquí que se trata de Justicia comata (Acanthaceae), especie ampliamente distribuida en América tropical desde el sur de México hasta Bolivia, Paraguay y el norte de la Argentina, en donde habita lugares con suelos anegados o muy húmedos (Ezcurra, 2019). Por este motivo, se subordina a $S$. beccabungoides como un nuevo sinónimo de $J$. comata.

\section{AGRADECIMIENTOS}

Se desea agradecer muy especialmente a Laurence Loze y Fred Stauffer (G) por el envío de las imágenes solicitadas. A Leonardo O. Alvarado-Cárdenas por el escaneado y el envío de la tesis doctoral de K. R. Gould. Por último, a los revisores anónimos y al Comité Editorial de Darwiniana, nueva serie, que ayudaron a mejorar considerablemente el manuscrito.

\section{BIBLIOGRAFÍA}

Braun, U. \& A.-K. Wittig. 2003. Typusmaterial des Herbariums der Martin-Luther-Universität Halle-Wittenberg (HAL). Teil 4: Taxa beschrieben von D. F. L. von Schlechtendal. Schlechtendalia 10: 15-65.

Bravo, L. D. 1971. Las especies argentinas de Spigelia (Loganiaceae). Darwiniana 16(3-4): 562-590.

Chamisso, L. K. A. von \& D. F. L. von Schlechtendal. 1826. De plantis in expeditione speculatoria Romazoffiana observatis. Plantaginaceae-Primulaceae. Linnaea 1(2): 165-226.

Chodat, R. H. 1901. Loganiaceae, en R. H. Chodat (ed.), Plantae Hasslerianae soit énumération des plantes récoltées au Paraguay par le Dr. Émile Hassler, d'Aarau (Suisse) de 1885-1895 et de 1898-1900 et déterminées par le Prof. Dr. R. Chodat avec l'aide de plusieurs collaborateurs. Bulletin de l'Herbier Boissier, sér. 2, 1: 407-408. DOI: https://doi. org/10.5962/bhl.title.45112
Chodat, R. H. \& E. Hassler. 1903. Plantæ Hasslerianae soit énumération des plantes récoltées au Paraguay par le Dr. Émile Hassler, d'Aarau (Suisse) de 1885 à 1902. seconde partie. Bulletin de l'Herbier Boissier, sér. 2, 3(10): 906-941.

Ezcurra, C. 2019. Acanthaceae, en F. O. Zuloaga \& M. J. Belgrano (eds.), Flora Vascular de la República Argentina 20: 21-40. Buenos Aires: Estudio Sigma S. R. L.

Fernández Casas, F. J. 2001. De neogaeis Spigeliis (Strychnaceæ) sparsae notulæ, 1-9. Fontqueria 55(5): 19-30.

Fernández Casas, F. J. 2016. El género Spigelia Linnaeus (1753). Nomenclatura. Adumbrationes ad Summae Editionem 70: 1-52.

Gould, K. R. 1997. Systematic studies in Spigelia. Ph.D. thesis, The University of Texas at Austin.

Hiepko, P. 1987. The collections of the Botanical Museum Berlin-Dahlem (B) and their history. Englera 7: 219-252.

Hurley, H. H. 1967. A taxonomic revision of the genus Spigelia (Loganiaceae). Ph.D. thesis, The George Washington University.

Islas-Hernández, C. S.; R. B. García \& L. O. AlvaradoCárdenas. 2017. New additions of Spigelia (Loganiaceae) in Mexico. Phytotaxa 331(2): 243-252. DOI: https://doi. org/10.11646/phytotaxa.331.2.8

Kraenzlin, F. W. L. 1908. II. Loganiaceæ austro-americanæ, en I. Urban (ed.), Plantæ novæ andinæ imprimis Weberbauerianæ III. Botanische Jahrbücher für Systematik, Pflanzengeschichte und Pflanzengeographie 40: 306-312.

Kraenzlin, F. W. L. 1916. XLVIII. Loganiaceae, en E. Hassler (ed.), Ex herbario Hassleriano: Novitates paraguarienses XXI. Repertorium Specierum Novarum Regni Vegetabilis 14: 292-295. DOI: https://doi.org/10.1002/fedr.19160141607

Peña Chocarro, M. C. \& J. De Egea. 2018. Checklist of endemic vascular plants of Paraguay. Phytotaxa 384(1): 1-47. DOI: https://doi.org/10.11646/phytotaxa.384.1.1

Popovkin, A. V.; K. G. Mathews, J. C. M. Santos, M. C. Molina \& L. Struwe. 2011. Spigelia genuflexa (Loganiaceae), a new geocarpic species from the Atlantic forest of northeastern Bahia, Brazil. PhytoKeys 6: 47-65. DOI: https://doi. org/10.3897/phytokeys.6.1654

Ramella, L.; P. Perret \& M. Soloaga. 2008. Catalogus Hasslerianus. Catálogo de las colecciones de Paraguay y regiones adyacentes hechas por Emil Hassler y otros colectores conservadas en el Conservatoire et Jardin botaniques de la Ville de Genève. Candollea 63: 282-287.

Stafleu, F. A. \& R. S. Cowan. 1976. Taxonomic literature. A selective guide to botanical publications and collections with dates, commentaries and types, Second edition, Volume I: A-G. Reg. Veg. 98, Bohn, Scheltema \& Holkema, Utrecht: [I]XVIII, 1-991. DOI: https://doi.org/10.5962/bhl.title.48631 
Stafleu, F. A. \& R. S. Cowan. 1979. Taxonomic literature. A selective guide to botanical publications and collections with dates, commentaries and types, Second edition, Volume II: H-Le. Reg. Veg. 98, Bohn, Scheltema \& Holkema, Utrecht: [I]-XVIII, 1-991. DOI: https://doi.org/10.5962/bhl. title. 48631

Struwe L., K. L. Gibbons, B. J. Conn, \& T. J. Motley. 2018. Loganiaceae, en Kadereit, J. \& V. Bittrich (eds.), Flowering Plants. Eudicots. The Families and Genera of Vascular Plants 15: 511-526. Springer. DOI: https://doi. org/10.1007/978-3-319-93605-5_8

Thiers, B. [permanentemente actualizado, consulta 2021] Index Herbariorum: a global directory of public herbaria and associated staff. New York Botanical Garden's Virtual Herbarium, http://sweetgum.nybg. org/ih
Turland, N. J.; J. H. Wiersema, F. R. Barrie, W. Greuter, D. L. Hawksworth, P. S. Herendeen, S. Knapp, W. -H. Kusber, D. -Z. Li, K. Marhold, T. W. May, J. McNeill, A. M. Monro, J. Prado, M. J. Price \& G. F. Smith (eds.). 2018. International Code of Nomenclature for algae, fungi, and plants (Shenzhen Code) adopted by the Nineteenth International Botanical Congress Shenzhen, China, July 2017. Regnum Vegetabile 159. Glashütten: Koeltz Botanical Books. DOI: https://doi.org/10.12705/Code.2018

Zanotti, C. A. 2020. Loganiaceae, en F. O. Zuloaga \& M. J. Belgrano (eds.), Flora Vascular de la República Argentina 19(1): 246-254. Buenos Aires: Estudio Sigma S. R. L.

Zuloaga, F. O.; M. J. Belgrano \& C. A. Zanotti. 2019. Actualización del Catálogo de las Plantas Vasculares del Cono Sur. Darwiniana, nueva serie 7(2): 208-278. DOI: https://doi.org/10.14522/darwiniana.2019.72.861 